\title{
Discrete Representation of Non-dominated Sets in Multi-objective Linear Programming
}

\author{
Lizhen Shao ${ }^{1, *}$, Matthias Ehrgott ${ }^{\mathrm{a}}$ \\ Key Laboratory of Advanced Control of Iron and Steel Process (Ministry of Education), School of Automation \\ and Electrical Engineering, University of Science and Technology Beijing, Beijing 100083, China \\ ${ }^{a}$ Department of Management Science, Lancaster University Management School, Bailrigg, Lancaster LA1 \\ $4 Y X$, United Kingdom
}

\begin{abstract}
In this paper we address the problem of representing the continuous but non-convex set of nondominated points of a multi-objective linear programme by a finite subset of such points. We prove that a related decision problem is NP-complete. Moreover, we illustrate the drawbacks of the known global shooting, normal boundary intersection and normal constraint methods concerning the coverage error and uniformity level of the representation by examples. We propose a method which combines the global shooting and normal boundary intersection methods. By doing so, we overcome their limitations, but preserve their advantages. We prove that our method computes a set of evenly distributed non-dominated points for which the coverage error and the uniformity level can be guaranteed. We apply this method to an optimisation problem in radiation therapy and present illustrative results for some clinical cases. Finally, we present numerical results on randomly generated examples.
\end{abstract}

Keywords: Multi-objective optimisation, linear programming, non-dominated set, discrete representation

\section{Introduction}

Multi-objective linear programming (MOLP) problems arise in many real world applications of Operations Research. Due to the presence of multiple conflicting objective functions, there is in general no single optimal value but an infinite continuous set of non-dominated objective function vectors. A non-dominated objective function vector (or non-dominated point) is the image $C x$ of an efficient solution $x$ of the MOLP. An efficient solution $x$ is a feasible solution of the MOLP such that there is no other feasible solution which is at least as good as $x$ in all objectives and strictly better in at least one.

\footnotetext{
* Corresponding author, phone number +861062332926

Email addresses: lshao@ustb.edu.cn (Lizhen Shao), m.ehrgott@lancaster.ac.uk (Matthias Ehrgott)
} 
In practical applications, a decision maker has to choose a most preferred point from the set of all non-dominated points. Since the non-dominated set of an MOLP with $p$ objectives is in general a set of dimension $p-1$, this is a difficult task. This is the motivation for computing a finite representative subset of non-dominated points: selecting from a finite set is easier and there is a wide variety of decision analysis methods for this task, see, e.g., Greco et al. (2016). Naturally, the representative set should be chosen in such a way that its cardinality is not too big, that each non-dominated point is close to at least one representative point (coverage error) and that the representative points are uniformly distributed and not too close to each other (uniformity level). We will give formal definitions (originally due to Sayin (2000)) in Section 2.

In this paper, which is an extension of an earlier conference paper (Shao and Ehrgott, 2007), we present a method to compute a finite representative subset of the non-dominated set of an MOLP. In Section 2 we formally define multi-objective linear programmes, review the definitions of the criteria for a good representation and point out a relationship to so called dispersion problems of location theory. We formulate the finite representation problem as a multi-objective optimisation problem over the non-dominated set of an MOLP and use the relationship to dispersion problems to prove that a decision version of our formulation is NP-complete. Section 3 is devoted to a review of the literature on methods to compute finite representations, with focus on the global shooting method (Benson and Sayin, 1997), the normal boundary intersection method (Das and Dennis, 1998) and the normal constraint method (Messac et al., 2003). We provide examples that show that they all may fail the coverage property or provide bounds on the uniformity level of the representation. Our own method, combining features of the global shooting and normal boundary intersection methods is described in Section 4. In Section 5 we prove bounds on the coverage error and the uniformity level of the representative set computed by our method under certain assumptions on the underlying MOLP. We also show that the method works in polynomial time in the bi-objective case. Section 6 provides some examples, presents an application of our method to an MOLP arising in radiotherapy treatment planning and provides numerical results on randomly generated examples. The numerical results provide empirical evidence for the theoretical results of the previous section. Section 7 summarises our contributions and identifies some questions for further research.

\section{Multi-objective Linear Programming and Finite Representation of a Set}

We shall use the following notation for the comparison of vectors $y^{1}$ and $y^{2} \in \mathbb{R}^{p}$. We write $y^{1} \leqq y^{2}$ if $y_{k}^{1} \leqq y_{k}^{2}$ for all $k=1, \ldots, p$. We use $y^{1} \leq y^{2}$ to indicate $y^{1} \leqq y^{2}$ but $y^{1} \neq y^{2}$, whereas $y^{1}<y^{2}$ means $y_{k}^{1}<y_{k}^{2}$ for all $k=1, \ldots, p$. We shall also use the notation $\mathbb{R}_{\prec}^{p}:=\left\{y \in \mathbb{R}^{p}: y \prec 0\right\}$ for $\prec \in\{\leqq, \leq,<\}$. 
A multiple objective linear programming problem (MOLP) in compact matrix notation can be written as the optimisation problem

$$
\min \{C x: x \in X\}
$$

where $C \in \mathbb{R}^{p \times n}$ is a $p \times n$ matrix of objective function coefficients, the rows $c_{k}{ }^{T}, k=1,2, \ldots, p$ of which are the coefficients of $p$ linear functions $c_{k}^{T} x, k=1,2, \ldots, p$. We assume that $X \subset \mathbb{R}^{n}$ is a nonempty compact polyhedral set of feasible solutions in decision space $\mathbb{R}^{n}$. The feasible set $Y$ in objective space $\mathbb{R}^{p}$ is defined by

$$
Y=\{C x: x \in X\}
$$

Since the image of a convex polyhedron under a linear map is also a convex polyhedron (see, e.g., Rockafellar (1970), it follows that $Y$ is also a nonempty compact convex polyhedron. In this paper, we shall make the further assumption that $Y_{N}$ contains at least two distinct points. Otherwise the non-dominated set is a singleton, and the MOLP (1) is trivial.

We next define efficient solutions and non-dominated points.

Definition 2.1. Feasible solution $x^{0} \in X$ is a (weakly) efficient solution to MOLP (1), if there does not exist any $x \in X$ such that $C x(<) \leq C x^{0}$. The set of all efficient solutions of MOLP (1) will be denoted by $X_{(W) E}$ and is called the (weakly) efficient set in decision space. Correspondingly, $y^{0}=C x^{0}$ is called a (weakly) non-dominated point and $Y_{(W) N}=\left\{C x: x \in X_{E}\right\}$ is the (weakly) non-dominated set in objective space for problem (1).

Theorem 2.2 is fundamental in multi-objective linear programming.

Theorem 2.2 (Isermann (1974)). Feasible solution $x^{0} \in X$ is an efficient solution of MOLP (1) if and only if there exists $a \lambda \in \mathbb{R}_{>}^{p}$ such that

$$
\lambda^{T} C x^{0} \leqq \lambda^{T} C x
$$

for all $x \in X$.

We recall that it is well known (see, e.g., Yu and Zeleny (1975); Naccache (1978)) that the non-dominated set $Y_{N}$ of an MOLP (1) is connected and consists of the union of faces of $Y$. It will be convenient to refer to maximal faces of $Y$ that belong to $Y_{N}$ as non-dominated faces.

Definition 2.3. Let $F \subset Y$ be a face of $Y$ such that $F \subseteq Y_{N}$ and $F$ is maximal with this property with respect to set inclusion. Then $F$ is called non-dominated face.

We shall now introduce the notion of representation of a set, the quality of such a representation, and the complexity of computing one. 
Let $Z \subset \mathbb{R}^{p}$ be a set and let $R \subset Z$ be a finite subset. $R$ is then called a discrete representation of $Z$. We are interested in "how well" $R$ represents $Z$. Sayin (2000) defines coverage, uniformity, and cardinality as the three attributes of quality of discrete representations. According to these three attributes, a good representation needs to contain a reasonable number of points, should not miss large portions of $Z$, and should not contain points that are very close to each other. This leads to the following definitions.

Definition 2.4. Let $\epsilon \geqq 0$ be a real number and $d$ be a metric. Representation $R$ of $Z$ is called an $\epsilon$-representation of $Z$ if for every $z \in Z$, there exists some $r \in R$ such that $d(z, r) \leqq \epsilon$.

Definition 2.5. Let $\delta \geqq 0$ be a real number and $d$ be a metric. Representation $R$ of $Z$ is called a $\delta$-uniform representation of $Z$ if

$$
\min _{r^{1}, r^{2} \in R, r^{1} \neq r^{2}}\left\{d\left(r^{1}, r^{2}\right)\right\} \geqq \delta .
$$

We note here, that throughout the paper we will always use the Euclidean distance as metric.

Sayin (2000) proposes measures to quantify the three quality attributes. The number of points contained in a representation obviously measures its cardinality. The coverage error $\epsilon$ and uniformity level $\delta$ are defined as follows.

The coverage error $\epsilon$ signifies how precisely the set $Z$ is being represented by the discrete representative subset $R$. It can be written as:

$$
\epsilon=\max _{z \in Z} \min _{r \in R} d(z, r)
$$

Thus, how well a fixed $z \in Z$ is covered is determined by the closest point to $z$ in the representation $R$. For the entire set $Z$, the coverage error depends on how well an arbitrary element of $Z$ is covered. Therefore, the coverage error $\epsilon$ is equal to the maximum coverage error for individual points in $Z$.

The coverage error is closely related to what we informally term the coverage property of a method to generate a discrete representation, namely the property, that every non-dominated point can potentially be selected as a representative point.

The uniformity level of representation $R$ can be measured by the distance between a pair of closest distinct points of $R$. Thus it can be expressed as

$$
\delta=\min _{r^{1}, r^{2} \in R, r^{1} \neq r^{2}} d\left(r^{1}, r^{2}\right)
$$

For a discrete representation, a small number of points, low coverage error, and high uniformity level are desirable.

From the discussion above, we can formally write the problem of finding a discrete representation of $Z$ as a multi-objective optimisation problem 


$$
\begin{array}{ll}
\min & |R| \\
\max & \min _{\left\{r^{i}, r^{j} \in R, r^{i} \neq r^{j}\right\}} d\left(r^{i}, r^{j}\right) \\
\min & \max _{z \in Z} \min _{r \in R} d(z, r) \\
\text { s.t. } & R \subset Z,|R|<\infty .
\end{array}
$$

Multi-objective optimisation problem $(4)-(7)$ is the problem of locating a small number of points in set $Z$ such that the points are far apart from each other, yet each point of $Z$ is close to at least one point of $R$. Such problems are known as dispersion problems in location theory. In the context of this paper, therefore, $Z$ would be $Y_{N}$, the non-dominated set of an MOLP.

This relationship between dispersion problems and multi-objective decision making was in fact first observed by White (1991): “... in the context of multiple objective problems, it may be that it is not only the maximal dispersion which is of interest, but also the representativeness of the solution obtained for the set $Z$ as a whole." However, he considers the problem

$$
\max \{\min \{d(x, y): x, y \in R\}: R \subseteq Z,|R|=p\}
$$

Thus, White (1991) considers only the uniformity measure, (5), keeps the cardinality constant and ignores the coverage error. He also restricts $Z$ to a finite set. More relevant is the work by Baur and Fekete (2001). They consider, amongst others, the so called pure dispersion problem

$$
\max \{\min \{d(v, w): v, w \in R\}: R \subset Z,|R|=p\}
$$

where $Z$ is a polygonal region in $\mathbb{R}^{2}$ and $d(v, w)$ is the geodetic distance between $v$ and $w$. They prove Theorem 2.6.

Theorem 2.6. Unless $P=N P$, there is no polynomial-time approximation scheme for pure geometric dispersion.

From this result it follows that the decision problem: Given a polygonal region $Z$, an integer $p$, and a constant $\delta$, does there exist a subset $R$ of $Z$ with cardinality $p$ such that $\min \{d(v, w): v, w \in$ $R\} \geqq \delta$ ? is NP-complete. Hence the canonical decision problem of the discrete representation problem stated in $(4)-(7)$, given $Z$, an integer $p$ and nonnegative scalars $\epsilon$ and $\delta$, does there exist a finite subset $R$ of $Y_{N}$ of cardinality $p$ such that the coverage error is at most $\epsilon$ and the uniformity level is at least $\delta$ is NP-complete, since it is NP-complete for the specific choice of $\epsilon$ such that $d\left(z^{1}, z^{2}\right) \leqq \epsilon$ for all $z^{1}, z^{2} \in Z$.

To relate this to the problem of finding discrete representations of the non-dominated set of an MOLP, we notice that, as mentioned in Section 1 the non-dominated set of an MOLP as 
defined in (1) is the union of finitely many non-dominated faces of $Y$. Therefore, finding discrete representations of $Y_{N}$ is equivalent to finding discrete representations of a union of polytopes.

To complement this hardness result, we notice that for discrete bi-objective optimisation problems Vaz et al. (2015) provide algorithms that solve the discrete representation problem for known $Y_{N}$ in time polynomial in $\left|Y_{N}\right|$ and $|R|$ for various combinations of coverage error, uniformity level, and $\epsilon$-indicator as quality measures. Eusébio et al. (2014) provide algorithms to compute $\delta$-uniform or $\epsilon$-representations for bi-objective integer network flow problems, but they do not analyse their complexity. In Section 4 we prove that our method computes a discrete representation of the non-dominated set of a bi-objective linear programme by solving $O(|R|)$ linear programmes without the knowledge of $Y_{N}$.

\section{A Survey of Existing Methods}

\subsection{Brief Literature Review}

We can categorise the methods for finding discrete representations of the non-dominated set of multi-objective optimisation problems into two groups, one is based on the knowledge of $X_{E}$ and the other works without the knowledge of $X_{E}$.

Based on the knowledge of $X_{E}$, Sayın (2003) proposes a procedure to find discrete representations with specified coverage errors. The procedure also specifies the uniformity level of the representations. Knowledge of $X_{E}$ can, however, not be assumed when solving a multi-objective optimisation problem. Therefore most methods work without the knowledge of $X_{E}$. A recent survey of methods for computing discrete representations in multi-objective optimisation can be found in Faulkenberg and Wiecek (2010). In the following, we focus on methods that are related to the concepts of the global shooting and the normal boundary intersection methods.

Benson and Sayin (1997) propose a global shooting method to find a representation of the nondominated set. This method has the coverage property, but it can not guarantee the uniformity, i.e. a bound on the uniformity level, of the representations it generates. We will discuss the global shooting method in more detail in Section 3.2. Das and Dennis (1998) propose a normal boundary intersection (NBI) method for finding non-dominated points for general (nonlinear) multi-objective optimisation problems. It uses the convex hull of the individual minima (CHIM) of the $p$ objectives as a reference plane. Evenly distributed reference points are placed on the CHIM and for each reference point a non-dominated point is computed by solving a single objective optimisation subproblem. While the method generates evenly distributed non-dominated points, some parts of the non-dominated set may be missed, i.e. it does not have the coverage property, a problem caused by the use of the CHIM. We will illustrate the method and its limitations in Section 3.3.

Based on the NBI and the $\epsilon$-constraint methods, Ismail-Yahaya and Messac (2002) propose the normal constraint $(\mathrm{NC})$ method. Instead of an equality constraint used in the subproblems 
of the NBI method, the NC method uses an inequality constraint to reduce the feasible set in the objective space of the multi-objective optimisation problem. Later, Messac et al. (2003) propose the normalised normal constraint (NNC) method. The NNC method works in a normalised objective space. However, both the NC and NNC methods have the same drawback as the NBI method, because they use the CHIM as reference plane. Realising this limitation of using the CHIM, Messac and Mattson (2004) improve the NNC method by using an extended CHIM instead of the CHIM as reference plane. However, uniformity level and coverage error of the discrete representation are not measured.

Martinez et al. (2007) improve the NNC method for bi-objective optimisation problems. The improvements are based on two types of techniques, i.e., nonlinear optimisation and genetic algorithms. Sanchis et al. (2008) propose a new alternative method, the enhanced normalised normal constraint (ENNC) method for multi-objective optimisation problems. They present the formulation of a new reference plane that improves the original normalised normal constraint method of Messac et al. (2003) using two approaches: a redefinition of the anchor points, i.e., vertices of the reference plane and an exact linear transformation between the objective space and the normalised objective space. Mueller-Gritschneder et al. (2009) propose a successive approach to systematically build up the representative non-dominated set. The approach is based on the generation of socalled trade-off limits. Motta et al. (2012) propose a novel modified procedure which is similar to the approach of Mueller-Gritschneder et al. (2009) and they claim that their method when applied to the NBI and NC methods for more than two objectives overcomes some of their deficiencies. Logist and Van Impe (2012) give theoretical insights in the conditions under which the NBI and the ENNC are able to generate the same candidate non-dominated points. Hancock and Mattson (2013) propose the smart normal constraint method for generating a "smart" non-dominated set. However, with the idea of smart they do not consider uniformity any more. Another recent paper dedicated to generating equidistant representations in bi-objective optimisation proposed by Faulkenberg and Wiecek (2012) is based on the $\epsilon$-constraint method.

It is worth mentioning that there are many approximation methods for multi-objective optimisation (the reader is referred to Ruzika and Wiecek (2005)) that compute some non-dominated points. However, since their goal is to approximate the whole non-dominated set and they do not aim at finding evenly distributed non-dominated points they may yield bad representations in terms of coverage error and uniformity level. Such methods are therefore not mentioned in the above survey.

We now present more details of some of the methods mentioned above. We note that although they are originally formulated for general multi-objective optimisation problems, we use MOLP notation throughout for consistency. 


\subsection{The Global Shooting Method}

As indicated in Section 1, we assume that the feasible set $Y$ in objective space is bounded. The global shooting method defines polyhedron $Y^{\prime}:=\left\{y \in \mathbb{R}^{p}: C x \leqq y \leqq \hat{y}\right.$ for some $\left.x \in X\right\}$, where $\hat{y}$ is chosen as a point so that for all $y \in Y_{N}$ it holds that $y \leqq \hat{y}$. For instance, $\hat{y}$ can be chosen as the anti ideal point $y^{A I}$ the components of which are defined by $y_{k}^{A I}:=\max \left\{y_{k}, y \in Y\right\}, k=1, \ldots, p$. Benson (1998) has shown that $Y^{\prime}$ has dimension $p$ and that $Y^{\prime}$ and $Y$ have the same non-dominated set.

The global shooting method then constructs a simplex $S$ that contains $Y^{\prime}$. A subsimplex $\hat{S}$ of $S$ (the non-dominated set of $S$ ) is chosen as the reference plane. Then, a discrete sample of reference points are placed on $\hat{S}$ and the method "shoots" from $\hat{y}$ towards each reference point as far as possible while remaining in $Y^{\prime}$. This is achieved by solving the single objective linear programme (the global shooting subproblem (8)) .

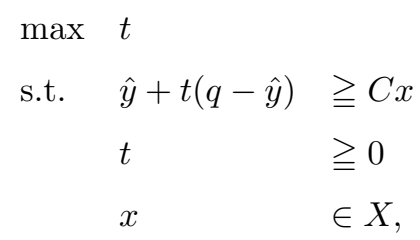

where $q$ is a reference point. Thus, a set of points on the boundary of $Y^{\prime}$ is computed and each reference point $q^{i}$ corresponds to a boundary point $y^{i}=\hat{y}+t_{i}(q-\hat{y})$ of $Y^{\prime}$, where $t_{i}$ is the optimal value of linear programme (8) for $q=q^{i}$. However, not every intersection point $y^{i}$ is non-dominated. Therefore it needs to be checked whether the intersection point $y^{i}$ is dominated or not by solving the following linear programme.

$$
\begin{array}{ll}
\min & \lambda^{T} y \\
\text { s.t. } & y \quad \leqq y^{i} \\
& y \quad \in Y^{\prime},
\end{array}
$$

where $\lambda \in \mathbb{R}^{p}$ is an arbitrary strictly positive vector. It is most convenient to choose $\lambda=e$, the vector of all ones. Point $y^{i}$ is non-dominated if and only if the optimal value of (9) is $\lambda^{T} y^{i}$. In case $y^{i}$ is dominated, an optimal solution to (9) is non-dominated and is added to the representative set in place of $y^{i}$.

Fig. 1 illustrates the global shooting method for an MOLP problem where $Y$ is the convex hull of vertices $(3,7),(2,9),(3,12),(6,11),(8,9),(9,7),(10,4)$ and $(6,5)$. The non-dominated extreme points of the MOLP are $(2,9),(3,7),(6,5)$ and $(10,4)$. There are 13 reference points on the reference plane which is the line segment from $(-2,12)$ to $(10,0)$. For each reference point, we solve problem (8) and obtain 13 intersection points with $Y^{\prime}$. Among the 13 intersection points, nine are 
non-dominated and four are dominated. The latter are shown as crosses in Fig. 1. After solving (9), the nine non-dominated intersection points are kept, whereas solving (9) for the four weakly non-dominated intersection points yields non-dominated point $y=(2,9)$. Thus the representative sets consist of the nine non-dominated intersection points and non-dominated point $(2,9)$.

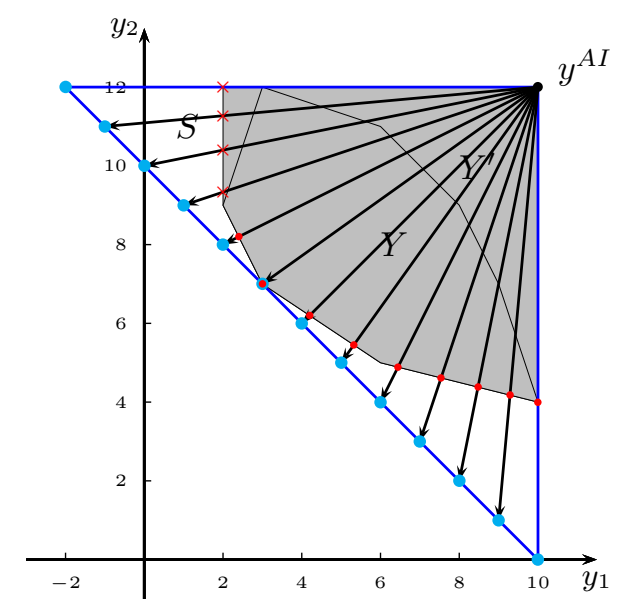

Figure 1: The global shooting method.

For the MOLP case, which is the topic of this paper, the global shooting method simply involves solving two linear programmes for each reference point. It has the coverage property because it puts reference points on $\hat{S}$ and $Y_{N} \subset \hat{S}+\mathbb{R}_{\geqq}^{p}$. We refer to Benson and Sayin (1997) for proofs of the properties of the global shooting method.

Next, we provide a series of MOLPs that shows that the uniformity level of the discrete representative set computed by the global shooting method can be arbitrarily small, even if the reference points are equidistantly distributed on the reference plane.

Example 3.1. Consider the MOLP problem $\min \left\{C x: A x \geqq b, x \in \mathbb{R}^{n}\right\}$ with

$$
C=\left(\begin{array}{ll}
1 & 0 \\
0 & 1
\end{array}\right), A=\left(\begin{array}{rr}
-1 & 0 \\
0 & -1 \\
M & 1 \\
0 & 1
\end{array}\right), b=\left(\begin{array}{r}
-M-1 \\
-M-1 \\
M^{2}+1 \\
1
\end{array}\right)
$$

where $M \geqq 1$.

Fig. 2 shows the feasible set $Y$ in objective space for $M=9$. The non-dominated set is the line segment from point $(M-1, M+1)$ to point $(M, 1)$. We use the global shooting method to obtain a set of non-dominated points. $M+2$ reference points which are evenly distributed on the reference plane (the line segment between $(0, M+1)$ and $(M+1,0))$ are used. They are $(0, M+1),(1, M),(2, M-1), \ldots,(M-1,2),(M, 1)$ and $(M+1,0)$. We obtain $M+1$ corresponding 
non-dominated points shown in Fig. 2. They are $(M-1, M+1),\left(\frac{M^{3}-M^{2}+M+1}{M^{2}+1}, \frac{M^{3}+M^{2}-M+1}{M^{2}+1}\right)$, $\ldots,\left(\frac{3 M^{2}-2 M-1}{3 M-1}, \frac{M^{2}+4 M-1}{3 M-1}\right)$ and $(M, 1)$. The Euclidean distance between the "first" two nondominated points $(M-1, M+1)$ and $\left(\frac{M^{3}-M^{2}+M+1}{M^{2}+1}, \frac{M^{3}+M^{2}-M+1}{M^{2}+1}\right)$ is $d_{1}=\frac{2}{\sqrt{M^{2}+1}}$ and the distance between the "last" two non-dominated points $\left(\frac{3 M^{2}-2 M-1}{3 M-1}, \frac{M^{2}+4 M-1}{3 M-1}\right)$ and $(M, 1)$ is $d_{2}=$ $\frac{(M+1) \sqrt{M^{2}+1}}{3 M-1}$ so that $d_{2}$ is equal to $\frac{(M+1)\left(M^{2}+1\right)}{6 M-2}$ times $d_{1}$. As $M$ approaches infinity, $d_{1}$ approaches zero, while $d_{2}$ approaches infinity. Therefore, as $M \rightarrow \infty$, the uniformity level tends to 0 and the coverage error tends to infinity. This clearly shows that the global shooting method cannot guarantee any positive uniformity level or any bounded coverage error.

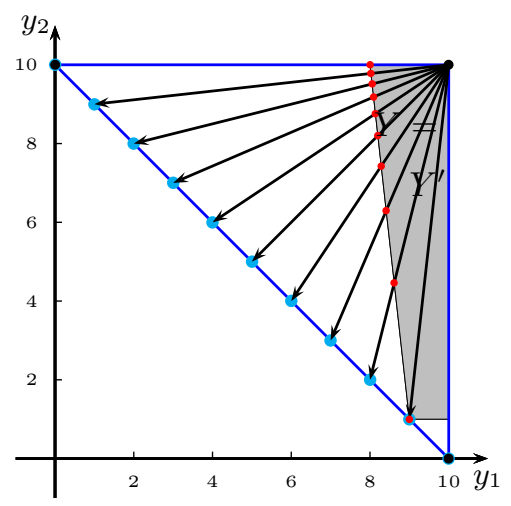

Figure 2: Unevenly distributed representative non-dominated points obtained by the global shooting method.

\subsection{The Normal Boundary Intersection (NBI) Method}

We first assume the individual minima of the functions $c_{k}^{T} x$ over $X$ are attained at $x^{k}$ for $k=1,2, \ldots, p$. Let $y^{k}=C x^{k}$ and let $y^{I}=\left(c_{1}^{T} x^{1}, c_{2}^{T} x^{2}, \ldots, c_{p}^{T} x^{p}\right)^{T}$ denote the ideal point. We note that in the original paper, Das and Dennis (1998) consider a multi-objective optimisation problem in which $Y$ is translated by $y^{I}$ so that the ideal point becomes 0 . To be consistent with the description of the other methods in this paper, we do not work with the translated problem.

The convex hull of the individual minima (CHIM) is then defined as the set of all convex combinations of the individual global minima of the objective functions, i.e. $\operatorname{conv}\left\{C x^{k}: k=\right.$ $1, \ldots, p\}$. A set of evenly distributed reference points on the CHIM is generated and for each of them a NBI subproblem is solved to find the farthest point on the boundary of $Y$ along the normal $\hat{n}$ of the CHIM pointing towards the ideal point. The NBI subproblem for a given reference point $q$ is the linear programme

$$
\begin{array}{lll}
\max & t & \\
\text { s.t. } & q+t \hat{n} & =C x \\
& t & \geqq 0 \\
x & \in X .
\end{array}
$$


Fig. 3 shows how the NBI method works for the same MOLP example with two objectives as in Fig. 1. For this example, all the points obtained are non-dominated and the non-dominated set is uniformly covered. For problems with more than two objectives, Das and Dennis (1998) state that the method may overlook portions of the non-dominated set. They claim that these overlooked areas are likely near the periphery of the non-dominated set. However, in Example 3.2 we provide an MOLP with $p=3$ objectives that shows that the normal to the CHIM may not always be positive and does therefore not find any non-dominated point on the largest part (any facet) of the non-dominated set. In fact, in this example, the NBI method only finds non-dominated points that belong to the CHIM. In other words, the NBI method does not have the coverage property.

Although Das and Dennis (1998) claim that the NBI method does compute evenly distributed non-dominated points, they do not provide bounds on the uniformity level of the computed representative set.

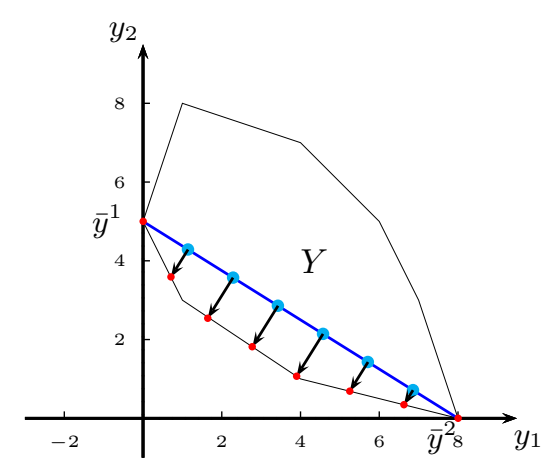

Figure 3: The NBI method.

\subsection{The Normal Constraint (NC) and Related Methods}

Like the NBI method, the normal constraint method (Ismail-Yahaya and Messac, 2002) also uses the CHIM (called the utopia plane in that paper) as the reference plane to put reference points. However, for each reference point, NC solves a different subproblem to compute a corresponding non-dominated point. The subproblem is described as follows.

$$
\begin{aligned}
& \min y_{p} \\
& \text { s.t. } \quad N_{k}^{T}(y-q) \leqq 0, \quad \text { for all } k=1, \ldots, p-1 \\
& y \quad \in Y \text {, }
\end{aligned}
$$

where $N_{k}=y^{p}-y^{k}$ and $y^{k}=C x^{k}$ is a vector such that $y_{k}^{k}$ is the minimal value of the $k$ th objective. 
The inequality constraint $N_{k}^{T}(y-q) \leqq 0$ reduces the feasible set in the objective space of the MOLP. Therefore, the subproblem is actually minimising the last single objective in the reduced feasible set in objective space subject to the reduced objective space.

Fig. 4 shows how the NC method works for the same MOLP example with two objectives as in Fig. 3.

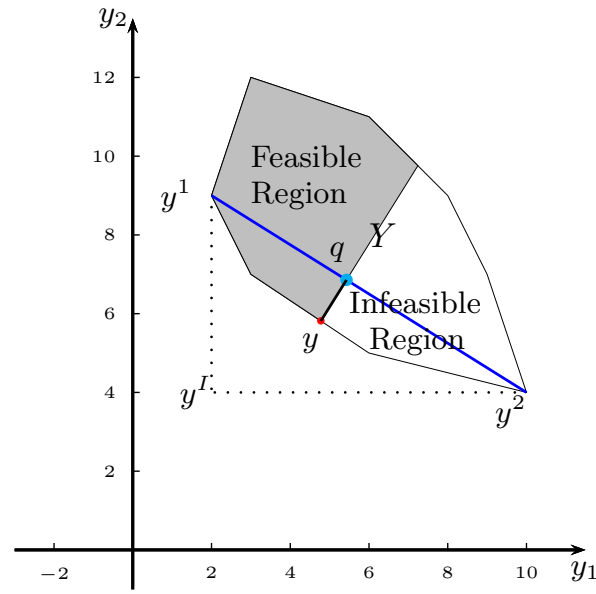

Figure 4: The NC method.

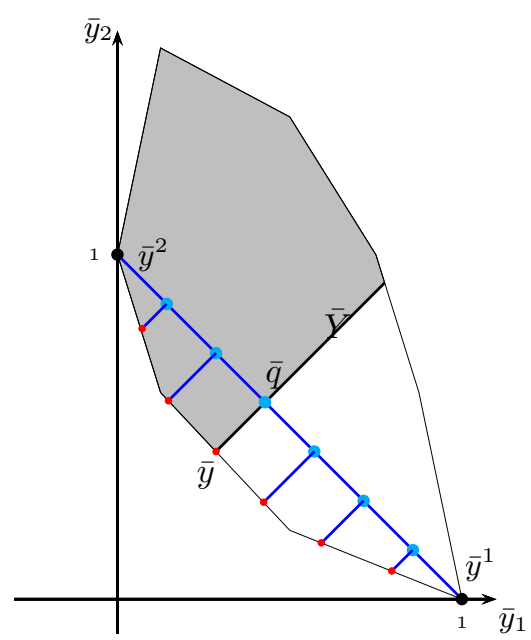

Figure 5: The normalised objective space of the NNC method.

For handling disparate objective magnitudes, scales, or ranges of objective function values for the different objective functions, Messac et al. (2003) propose the normalised normal constraint (NNC) method. The NNC method is the same as the NC method, except that the subproblems are formulated in a normalised objective space, see Figure 5.

Similar to the NBI method, the NC and NNC methods do not provide a guarantee that the generated set of non-dominated points will represent the non-dominated set well if $p \geqq 3$. Realising this limitation, Messac and Mattson (2004) improve the NC method by using an extended CHIM instead of the CHIM as reference plane. They claim that this improvement will generate evenly spread non-dominated points over the entire non-dominated set. However, as Example 3.2 shows, this is not necessarily always the case. Messac and Mattson (2004) also generalise the subproblem. Instead of minimising the last single objective, the subproblem can minimise any single objective. Messac and Mattson (2004) expect that the same result is obtained no matter which objective is minimised. However, this is not always true. Example 3.2 can be used to show that solving different subproblem gives different results.

Example 3.2. We consider the linear relaxation of a binary assignment problem with three objectives. The cost coefficients of the three linear objective functions are shown as matrices $c_{1}, c_{2}$ 
and $c_{3}$,

$$
c_{1}=\left(\begin{array}{llll}
3 & 6 & 4 & 5 \\
2 & 3 & 5 & 4 \\
3 & 5 & 4 & 2 \\
4 & 5 & 3 & 6
\end{array}\right), c_{2}=\left(\begin{array}{cccc}
2 & 3 & 5 & 4 \\
5 & 3 & 4 & 3 \\
5 & 2 & 6 & 4 \\
4 & 5 & 2 & 5
\end{array}\right), c_{3}=\left(\begin{array}{cccc}
4 & 2 & 4 & 2 \\
4 & 2 & 4 & 6 \\
4 & 2 & 6 & 3 \\
2 & 4 & 5 & 3
\end{array}\right) .
$$

We define $Y^{\prime}:=\left\{y \in \mathbb{R}^{3}: C x \leqq y \leqq \hat{y}\right.$ for some $\left.x \in X\right\}$ and choose $\hat{y}$ as $(21,21,21)$, which is greater than the anti-ideal point $(20,20,20)$ in this example. Fig. 6 shows $Y^{\prime}$ as the convex hull of the 16 extreme points $(21,21,21),(11,21,21),(21,9,21),(21,21,10),(11,11,14),(11,11$, 21), (15, 9, 21), (11, 21, 14), (21, 14, 10), (19, 21, 10), (13, 21, 11), (19, 14, 10), (21, 9, 17), (21, $11,14),(15,9,17),(13,16,11)$.

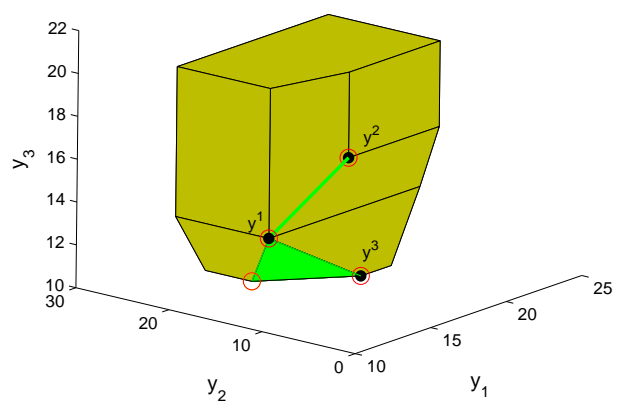

Figure 6: $Y^{\prime}$ and the non-dominated set in Example 3.2.

The four points $y^{1}=(11,11,14), y^{2}=(15,9,17), y^{3}=(19,14,10)$ and $(13,16,11)$ represented by circles are the non-dominated extreme points of $Y^{\prime}$. The non-dominated set consists of a line segment from point $y^{1}$ to point $y^{2}$ and a triangular facet which is the convex hull of $y^{1}, y^{3}$ and $(13,16,11)$.

The three dots at $y^{1}, y^{2}$ and $y^{3}$ in Fig. 6 are the unique non-dominated points at which the individual minima of the three objectives are attained. Hence the CHIM is the convex hull of these three points. The normal of the CHIM is $\hat{n}=(1,-40,-28)$ or $\hat{n}=(-1,40,28)$, which are not positive. Placing evenly distributed reference points on the CHIM, the results of the NBI, $\mathrm{NC}$ and NNC methods are shown in Fig. 7, 8 and 9, respectively. In those figures, circles represent the reference points, while dots represent non-dominated points generated by the respective methods. It is worth noting here, that neither Das and Dennis (1998) nor Messac et al. (2003) specify procedures for checking the non-dominance of points generated by solving subproblems (10) and (11), respectively. However, in this example, the optimal solutions of both subproblems for reference points located on the CHIM are those reference points themselves. For testing the methods, we did, therefore, implement the same non-dominance check that we use for the RNBI 
method described in Section 4 below.

We did not obtain any non-dominated points in the interior of the non-dominated facet by any of the three methods. For the NBI method, this occurs because the angle between the nondominated facet and the CHIM is approximately 151.5 > 90 degrees. But even for the NC and NNC method, the subproblems only generate non-dominated points on the (relative) boundary of the non-dominated facet and on the non-dominated edge between $y^{1}$ and $y^{2}$. Hence, all three methods clearly miss the major portion of the non-dominated set.

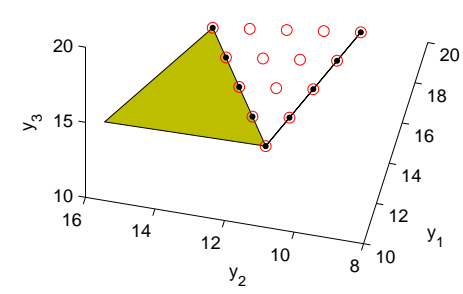

Figure 7: Reference points and nondominated points generated by the NBI method in Example 3.2.

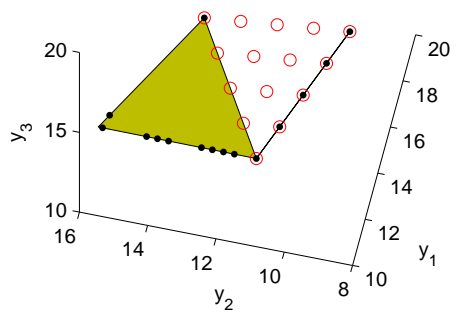

Figure 8: Reference points and nondominated points generated by the NC method in Example 3.2.

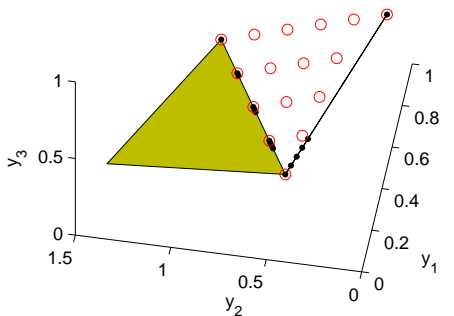

Figure 9: Reference points and nondominated points generated by the NNC method in Example 3.2.

We also applied the improved NC and NNC methods with extended CHIM. We chose the extended reference plane big enough so that the projection of the non-dominated set is contained in the plane. For the NC method, we also solved different subproblems for the same set of reference points to see if they yield the same result or not, they didn't. We omit detailed results and figures, but note that all generated non-dominated points are on the boundary of the non-dominated set.

While Benson and Sayin (1997) have shown that the global shooting method has the coverage property, Example 3.2 shows that the NBI and NC methods do not have this property. This result indicates, that in general these methods will not be able to compute $\epsilon$-representations for arbitrary $\epsilon$.

\section{The Revised Normal Boundary Intersection Method}

As discussed in Section 3.2 above, the global shooting method satisfies the coverage property, and the NBI method (Section 3.3) can generate evenly distributed non-dominated points. Therefore, we propose a revised NBI method which combines these two approaches. The revised normal boundary intersection (RNBI) method provides a priori guarantees on both the uniformity level and coverage error. 
On the one hand, instead of the CHIM, the RNBI method uses the non-dominated subsimplex $\hat{S}$ of the simplex $S$ that is used in the global shooting method (Benson and Sayin, 1997) as the reference plane on which to put equidistant reference points. By doing this, it retains the coverage property. On the other hand, by solving single objective subproblems similar to (10), the RNBI method generates a set of non-dominated points with a known positive bound on the uniformity level.

The RNBI method involves choosing a reference plane, placing equidistant reference points on the plane and computing the intersection points of rays emanating from the reference points in a direction normal to the reference plane with the feasible set $Y$ in objective space. Finally, the non-dominance of the computed intersection points is checked by solving a linear programme. In the following paragraphs we explain each of these steps in detail.

Constructing the Reference Plane. The reference plane is the same as in the global shooting method of Benson and Sayin (1997). For completeness, we describe the details of this construction here.

First, let

$$
\beta:=\min \left\{e^{T} y: y \in Y\right\}
$$

where $e \in \mathbb{R}^{p}$ is a vector in which each entry is 1 .

Next, we define $p+1$ points $v^{k} \in \mathbb{R}^{p}$, for $k=0,1, \ldots, p$. Let $v^{0}=y^{A I}$ and, for $k=1,2, \ldots, p$ and $l=1,2, \ldots, p$, let

$$
v_{l}^{k}= \begin{cases}y_{l}^{A I}, & \text { if } l \neq k \\ \beta+y_{k}^{A I}-e^{T} v^{0}, & \text { if } l=k\end{cases}
$$

As Benson and Sayin (1997) have shown, the convex hull $S$ of $\left\{v^{k}: k=0,1, \ldots, p\right\}$ is a $p$-dimensional simplex, and $S$ contains $Y$.

The subsimplex of $S$ given by the convex hull $\hat{S}$ of $\left\{v^{k}: k=1,2, \ldots, p\right\}$ is the non-dominated set of $S$. It is contained in the hyperplane $\left\{y \in \mathbb{R}^{P}: e^{T} y=\beta^{*}\right\}$, where $\beta^{*}$ is the optimal value of (12). This hyperplane supports $Y$ and $Y_{N}$ at all optimal solutions of (12).

Placing Equidistant Points on the Reference Plane. Clearly, for $p=2, \hat{S}$ is a line segment. Proposition 4.1 shows that in the general case of $p>2$ objectives, $\hat{S}$ is a $p-1$ dimensional simplex with equal edge length.

Proposition 4.1. The reference plane $\hat{S}$ is an equilateral simplex.

Proof. Let us consider two vertices $v^{i}$ and $v^{j}, i, j \in\{1, \ldots, p\}$ and assume without loss of generality 
that $i<j$.

$$
\begin{aligned}
v^{i}-v^{j}= & \left(v_{1}^{i}, \ldots, v_{i}^{i}, \ldots, v_{j}^{i}, \ldots, v_{p}^{i}\right)^{T}-\left(v_{1}^{j}, \ldots, v_{i}^{j}, \ldots, v_{j}^{j}, \ldots, v_{p}^{j}\right)^{T} \\
= & \left(y_{1}^{A I}, \ldots, \beta+y_{i}^{A I}-e^{T} v^{0}, \ldots, y_{j}^{A I}, \ldots, y_{p}^{A I}\right)^{T}- \\
& \left(y_{1}^{A I}, \ldots, y_{i}^{A I}, \ldots, \beta+y_{j}^{A I}-e^{T} v^{0}, \ldots, y_{p}^{A I}\right)^{T} \\
= & \left(0, \ldots, \beta+y_{i}^{A I}-e^{T} v^{0}-y_{i}^{A I}, \ldots, y_{j}^{A I}-\left(\beta+y_{j}^{A I}-e^{T} v^{0}\right), 0 \ldots, 0\right)^{T} \\
= & \left(0, \ldots, \beta-e^{T} v^{0}, \ldots,-\beta+e^{T} v^{0}, \ldots, 0\right)
\end{aligned}
$$

Thus the distance between any two vertices of $\hat{S}$ is equal to $d\left(v^{i}, v^{j}\right)=\sqrt{2}\left(\beta-e^{T} v^{0}\right)$.

For $p=3$, Fig. 10 shows how a triangular lattice can be used to generate equidistant points on $\hat{S}$. In the general case of $p$ objectives, the $i$ th reference point $q^{i}$ is given by

$$
q^{i}=\sum_{k=1}^{p} \alpha_{k}^{i} v^{k}
$$

where $0 \leqq \alpha_{k}^{i} \leqq 1$ and $\sum_{k=1}^{p} \alpha_{k}^{i}=1$. By varying $\alpha_{k}^{i}$ from 0 to 1 with a fixed increment of $\eta_{k}$ a finite set of equidistant points on the reference plane can be generated. For the three objective case in Fig. $10 \eta_{k}=0.25$. We denote $d s$ the distance between two closest reference points.

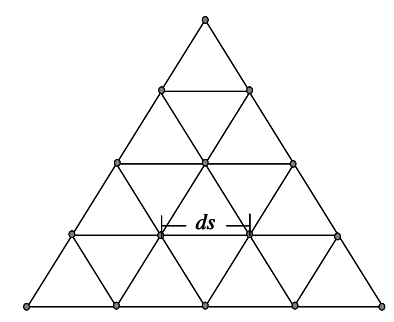

Figure 10: Equidistant reference points on the reference plane.

Computing the Intersection Points. Given a reference point $q$ on $\hat{S}$, the RNBI subproblem searches for the point in $Y$ that is closest to the reference point along the normal direction $e$. This is achieved by solving the RNBI subproblem (14).

$$
\begin{array}{lll}
\min & t & \\
\text { s.t. } & q+t e & \in Y \\
& t & \geqq 0 .
\end{array}
$$

Fig. 11 illustrates the RNBI method for the same MOLP example that was used in Figs. 1 and 3 to illustrate the global shooting and the NBI method. The reference plane and the placement of reference points are the same as for the global shooting method. However the search for intersection 
points with $Y$ uses a direction normal to the reference plane, as in the NBI method. Since due to our assumption of boundedness of $Y$ (and therefore $S$ ), the linear programme (14) cannot be unbounded, it is either infeasible or has an optimal solution. In fact, there are three scenarios for the solution of (14), see also Fig. 11.

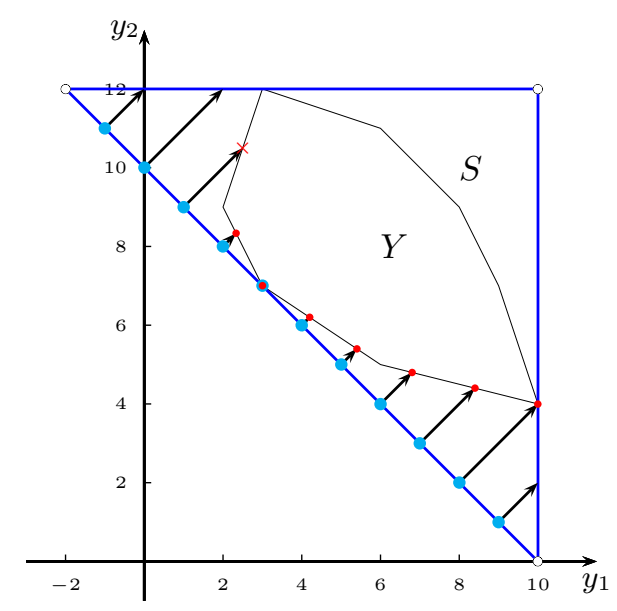

Figure 11: The revised normal boundary intersection method.

1. (14) is infeasible. This occurs if and only if there is no intersection between the ray $\{q+t e$ : $t \geqq 0\}$ and $Y$.

2. (14) has an optimal solution $t^{*}$ if and only if the ray $\{q+t e: t \geqq 0\}$ and $Y$ intersect. In this case, $y^{*}=q+t^{*} e$ belongs to the boundary of $Y$. Two subcases may occur.

(a) The intersection point $y^{*}$ is dominated.

(b) The intersection point $y^{*}$ is non-dominated.

Because in the case that (14) has an optimal solution, the identified intersection point $y^{*}$ may or may not be non-dominated it is necessary to check the status of $y^{*}$. A simple non-dominance filter can be applied to the set of all generated intersection points to eliminate some of them, see Messac et al. (2003). This method has the advantage of being fast, but it may accept some dominated points (often ones close to $Y_{N}$ ) as non-dominated.

An exact method to test the non-dominance of an intersection point $y^{*}$ is similar to (9) and is provided by Proposition 4.2, a proof of which can be found in Ehrgott (2005).

Proposition 4.2. Let $y^{*} \in Y$ and let $\lambda \in \mathbb{R}_{>}^{p}$. Then $y^{*} \in Y_{N}$ if and only if $y^{*}$ is an optimal solution to the linear programme (15),

$$
\begin{array}{ll}
\min & \lambda^{T} y \\
\text { s.t. } & y \leqq y^{*} \\
& y \in Y .
\end{array}
$$




\section{Properties of the RNBI Method}

In this section we investigate the properties of the RNBI method, in particular we prove bounds on the uniformity level and the coverage error of the representative set generated by the RNBI method.

First of all, we notice that for every non-dominated point $\hat{y}$ of the MOLP (1) there is a point $\hat{q} \in \hat{S}$ such that (14) has an optimal solution $\hat{t}$ such that $\hat{q}+\hat{t} e=\hat{y}$. This point is simply the projection in direction $e$ of $\hat{y}$ onto $\hat{S}$. Hence it follows, similar to Benson and Sayin (1997) for the global shooting method, that the RNBI method has the coverage property. Next we shall investigate the uniformity level.

Let $F$ be a non-dominated facet of $Y$ with normal $\hat{n}$. Since the normal of $\hat{S}$ is $e$ by definition, the angle between $\hat{S}$ and $F$ is given by

$$
\cos \theta=\frac{e^{T} \hat{n}}{\|e\|\|\hat{n}\|}=\frac{\hat{n}_{1}+\cdots+\hat{n}_{p}}{\sqrt{\left(\hat{n}_{1}\right)^{2}+\cdots+\left(\hat{n}_{p}\right)^{2}} \sqrt{p}} .
$$

Since, according to Theorem 2.2 and Definition 2.3 the normal $\hat{n}$ to $F$ is an element of $\mathbb{R}_{>}^{p}$ we have

$$
\frac{\hat{n}_{1}+\cdots+\hat{n}_{p}}{\sqrt{\left(\hat{n}_{1}\right)^{2}+\cdots+\left(\hat{n}_{p}\right)^{2}} \sqrt{p}}>\frac{\hat{n}_{1}+\cdots+\hat{n}_{p}}{\sqrt{\left(\hat{n}_{1}+\cdots+\hat{n}_{p}\right)^{2}} \sqrt{p}}=\frac{1}{\sqrt{p}} .
$$

Therefore

$$
\frac{1}{\sqrt{p}}<\cos \theta \leqq 1
$$

and $\theta$ is in the range of $0 \leqq \theta<\arccos \frac{1}{\sqrt{p}}$.

For $p=2,0 \leqq \theta<\frac{\pi}{4}$ and for $p=3,0 \leqq \theta<\arccos \frac{\sqrt{3}}{3}$, i.e., the range of angles between the reference plane $\hat{S}$ and non-dominated facets increases with $p$.

Now for the equidistant reference points with distance $d s$ that the RNBI method places on $\hat{S}$ it follows that the corresponding non-dominated points in the representative set have a distance of $d s / \cos \theta<\sqrt{p} d s$.

Fig. 12 shows an example with two objectives $(p=2)$ so that non-dominated facets are line segments. $F_{1}$ is a non-dominated facet, while $F_{2}$ is a weakly non-dominated facet. Angles between non-dominated facets and the reference plane must be smaller than $\frac{\pi}{4}$, whereas the angle between the reference plane and the weakly non-dominated facet $F_{2}$ is $\theta=\frac{\pi}{4}$. The distance between two closest non-dominated points obtained by the RNBI method is between $d s$ and $\sqrt{2} d s$.

Since for an MOLP (1) with $p$ objectives we have shown that for the distance $d$ between two closest non-dominated points in the representative set it holds that $d s \leqq d<\sqrt{p} d s$, we have $\min _{r^{1}, r^{2} \in R, r^{1} \neq r^{2}}\left\{d\left(r^{1}, r^{2}\right) \geqq d s\right.$. Thus, Theorem 5.1 is proved.

Theorem 5.1. Let $R$ be the representative subset of $Y_{N}$ obtained by the RNBI method. Then $R$ is a ds-uniform representation of $Y_{N}$. 


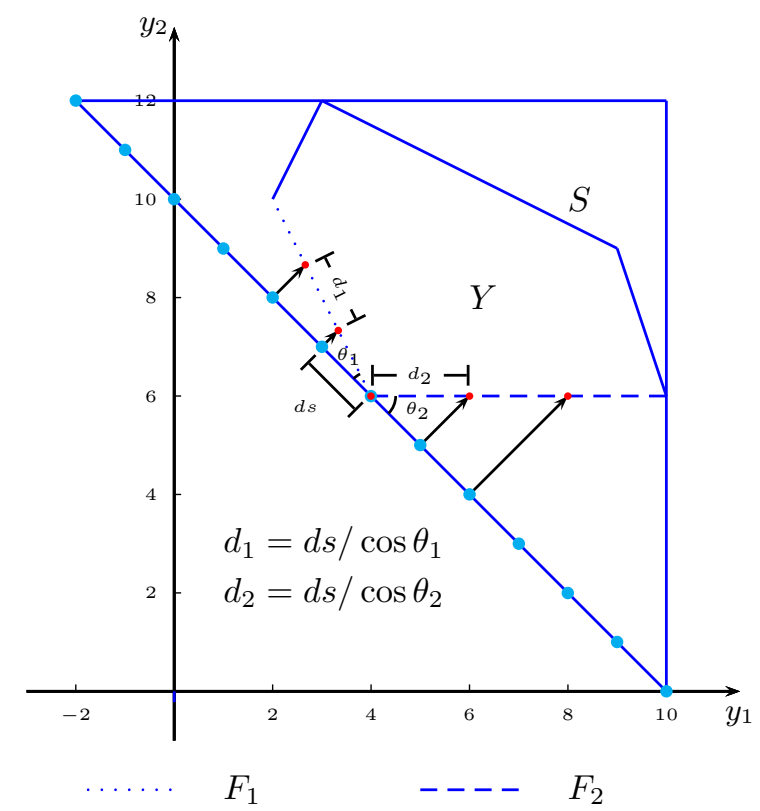

Figure 12: Analysis of distances for a 2D example.

The width $w(B)$ of a convex set $B \subset \mathbb{R}^{p}$ is defined as the smallest Euclidean distance between two supporting hyperplanes of $B$. Since this means that the width of any convex set of dimension less than $p$ is 0 but we want to measure the width of projections of subsets of $Y_{N}$ onto $\hat{S}$, we also define the width of a convex set on a hyperplane $\hat{S}, w_{\hat{S}}(B)$ as the minimal distance between two parallel supporting hyperplanes perpendicular to $\hat{S}$.

It is well known (Yu and Zeleny, 1975), that the non-dominated set of MOLP (1) is the finite union of maximal non-dominated faces of $Y$, i.e. $Y_{N}=\cap_{k=1}^{K} F_{k}$. Now, let $Y_{N}^{p}$ be the projection of $Y_{N}$ to the reference plane $\hat{S} . Y_{N}^{p}$ can then be written as the union of $K$ polytopes $\bigcup_{k=1}^{K} O_{k}$ on $\hat{S}$, where $O_{k}$ is the projection of $F_{k}$ onto $\hat{S}$. Using this notion, we can now prove the bound of $\sqrt{p} d s$ on the coverage error of the discrete representation generated by the RNBI method under an assumption on the width of sets $O_{k}$.

Theorem 5.2. Let $d s$ be the distance between reference points and assume that each set $O_{k}$ in the projection $Y_{N}^{p}$ of $Y_{N}$ satisfies $w_{\hat{S}}\left(O_{k}\right) \geqq d s$, then the representative set $R$ obtained by the revised NBI method is a $\sqrt{p} d s$-representation of $Y_{N}$.

Proof. Let $Q$ be the set of reference points for which solving (14) results in the set of representative points $R$. Since the width of $O_{k}, k=1 \ldots, K$ on the reference plane $\hat{S}$ is greater than or equal to $d s$, it follows that $Q \cap O_{k} \neq \emptyset$ for all $k=1, \ldots, K$.

Let $y \in Y_{N}$ and let $o$ be the projection of $y$ to $\hat{S}$. Clearly $o \in O_{k}$ for some $k \in\{1, \ldots, K\}$. Therefore, there must exist $q \in Q \cap O_{k}$ such that $d(o, q) \leqq d s$ because the distance of reference points is $d s$. Clearly, there also exists $r \in R$ which is the representative point generated by $q$. 
From the analysis of distances between representative points, it follows that $d(y, r) \leqq \sqrt{p} d s$

Therefore, for any non-dominated point $y \in Y_{N}$, there must exist $r \in R$ such that $d(y, r) \leqq$ $\sqrt{p} d s$

We remark that the proof of Theorem 5.2 also shows that, even in the case that not all $O_{k}$ have width at least $d s$, any non-dominated facet of $Y_{N}$ that satisfies the condition $w_{\hat{S}}\left(O_{k}\right) \geqq d s$ will be present in the representation. Hence it implies that only non-dominated faces that are "too small" because either their dimension is smaller than $p-1$ or their width is smaller than $\sqrt{p} d s$ can be missed.

Theorems 5.1 and 5.2 quantify the quality of the discrete representation generated by the RNBI method in terms of coverage error and uniformity level. They also clarify that the only parameter of the method is the distance $d s$ between two closest reference points. Since $\hat{S}$ is an equilateral simplex, this then determines the number of reference points and hence an (as we shall see very weak) upper bound on the cardinality of the discrete representation, the lower bound $d s$ on the uniformity level, and the the upper bound $\sqrt{p} d s$ on the coverage error. By increasing $d s$, the cardinality will increase, the uniformity level will decrease and the coverage error will decrease. We emphasise that the RNBI method does not allow independent control of the three measures of quality of the discrete representation. Nevertheless, it is to the best of our knowledge the first method that allows the computation of a discrete representation of the non-dominated sets of MOLPs with guaranteed coverage error and uniformity level, and without the knowledge of $Y_{N}$.

Finally, we consider the special case of MOLPs with $p=2$ objectives. Let $y^{\text {lex(1,2) }}$ and $y^{\text {lex }(2,1)}$ denote the two lexicographically optimal non-dominated points and $o^{\operatorname{lex}(1,2)}$ and $o^{\text {lex }(2,1)}$ their projections on the reference plane. Then for any reference point $q$ such that $q_{1}<o_{1}^{l e x(1,2)}$ or $q_{1}>o_{1}^{l e x(2,1)}$ we have that $(14)$ is either infeasible or its optimal solution yields a dominated point. On the other hand, all reference points with $o_{1}^{l e x(1,2)} \leqq q_{1} \leqq o_{1}^{l e x(2,1)}$ yield non-dominated points. Hence, to compute a representation $R$ of cardinality $|R|=r$ one places $r$ equally spaced reference points on the line segment from $o^{l e x(1,2)}$ to $o^{l e x(2,1)}$ and solve (14) for these reference points. The resulting representation will have coverage error $\sqrt{2} \delta$ and uniformity level $\delta$, where

$$
\delta=d\left(o^{l e x(1,2)}, o^{l e x(2,1)}\right) /(r-1) .
$$

Theorem 5.3. For any MOLP with $p=2$ objectives, such that $Y$ is bounded, the RNBI method computes a $\delta$-uniform $\sqrt{2} \delta$-representation of cardinality $r$ for $Y_{N}$ in time polynomial in the size of the MOLP and linear in $r$.

\section{Examples and Numerical Results}

In this section, we first apply the RNBI method to Examples 3.1 and 3.2, which we used before to illustrate that the global shooting and NBI methods cannot provide a guarantee on the 
uniformity level or the coverage error. Then, we present an application of the RNBI method to the beam intensity optimisation problem arising in the planning of radiotherapy treatment of cancer. Finally, we present the results of numerical tests of the RNBI method on randomly generated linear programmes with between three and eight objectives.

In Example 3.1 we apply the RNBI method with the the same set of reference points as we used for the global shooting method. The RNBI method generates six non-dominated points as shown in Fig. 13. These six points are evenly distributed on the non-dominated set and the distance between the two closest points in the representation is 1.9079. The guaranteed uniformity level in this example is of course $\sqrt{2}$. According to Theorems 5.1 and 5.2, the distance between two closest representative points is at least $\sqrt{2}$ and at most $\sqrt{2} \cdot \sqrt{2}=2$. Hence the RNBI method computes a $\sqrt{2}$-uniform 2-representation of the non-dominated set.

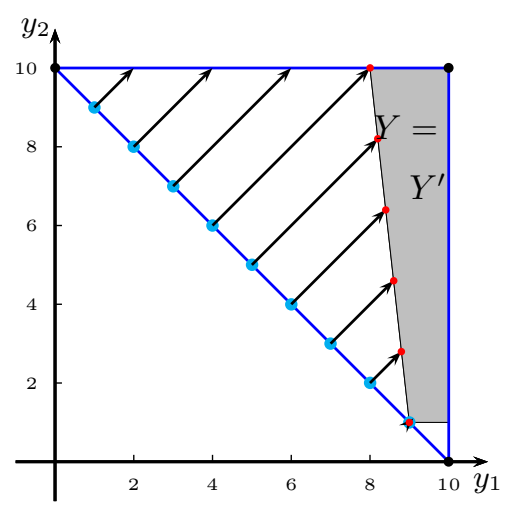

Figure 13: The non-dominated points generated by the RNBI method for Example 3.1.

Next, we apply the RNBI method to Example 3.2. We use reference points with distance $\sqrt{2}$ as in the previous example. This results in 325 reference points. The RNBI method generates 33 intersection points, ten of which are non-dominated points. These are shown in Fig. 14. We note that all non-dominated points generated by the method are on the non-dominated facet defined by vertices $(11,11,14),(19,14,10)$ and $(13,16,11)$. No non-dominated point on the edge between $(11,11,14)$ and $(15,9,17)$ is generated. This is of course expected due to Theorem 5.2 , because the width of the projection of the non-dominated edge onto the reference plane is equal to 0 . Thus, the RNBI method correctly computes a representation of the non-dominated facet, without guaranteeing any coverage of the non-dominated edge.

We shall now apply the RNBI method to a multi-objective linear programme derived from the so-called beam intensity optimisation problem in the planning of radiation therapy treatment for cancer. This serves to illustrate the potential of the RNBI method in practical application. We refer the reader to Ehrgott et al. (2009) for more details on optimisation problems in this domain. The goals of radiation therapy are to deliver a uniform dose as close as possible to a 


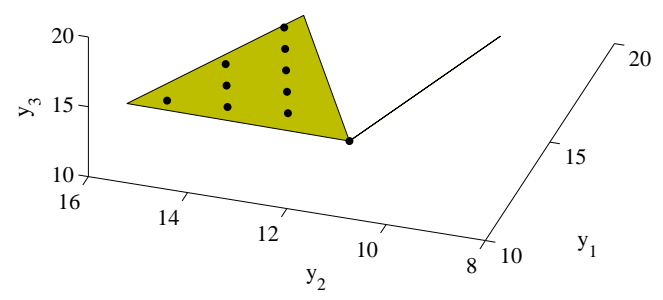

Figure 14: The set of non-dominated points generated by the RNBI method for Example 3.2.

prescribed dose to the planning target volume (which contains the tumour to be treated), while at the same time sparing the surrounding normal tissues and organs at risk as much as possible from the harmful effects of radiation. Given the number of beams and beam directions from which to irradiate the patient, beam intensity optimisation computes beam intensity profiles that yield the best dose distribution for treating a particular patient under consideration of clinical and physical constraints. Due to the conflicting goals, the beam intensity optimisation problem can, for example, be formulated as an MOLP in the form provided in Shao and Ehrgott (2008). In this MOLP the objectives are to minimise the maximum deviations $\alpha, \beta, \gamma$ of delivered dose from prescribed lower bounds on the dose delivered to the tumour and from prescribed upper bounds on the dose delivered to the organs at risk and other normal tissue, respectively.

We use three clinical cases, an acoustic neuroma $(\mathrm{AC})$, a prostate tumour $(\mathrm{PR})$ and a pancreatic lesion (PL). These are ordered according to the number of constraints and the number of variables. The RNBI method was implemented in Matlab 7.3 using the Matlab optimisation toolbox as LP solver and all tests were run on a PC with $2.5 \mathrm{GHz}$ processor speed and 4.0 GB RAM. In Table 1 , we list the number of reference points (RP), the number of intersection points generated (IP), as well as the number of non-dominated points (NP). The fourth column shows the distance $d s$ between two closest reference points. Finally, the CPU time in seconds is shown. For each case, we used two different numbers of reference points.

We notice that for all three cases, more than half of the reference points did not generate intersection points. However, checking infeasibility of (14) was not time consuming in this application, but this may be an issue for other MOLP problems. Moreover, Table 1 shows that for the PR and PL cases not every intersection point is non-dominated. Hence, checking non-dominance by solving linear programme (15) is necessary, but may be more time consuming than checking infeasibility. Clearly, the main effort in applying the RNBI method is in solving LPs. Hence, the time taken depends on the number of reference points, since at least one (but at most two) LPs 
need to be solved for each reference point.

Table 1: The number of reference points, intersection points with $Y$, non-dominated points, distance between closest reference points and CPU time in seconds for the application of the RNBI method to an MOLP in radiotherapy treatment planning.

\begin{tabular}{lrrrrr}
\hline & $\mathrm{RP}$ & $\mathrm{IP}$ & $\mathrm{NP}$ & $d s$ & $\mathrm{CPU}$ \\
\hline $\mathrm{AC}$ & 378 & 72 & 72 & 1.04 & 23.828 \\
$\mathrm{PR}$ & 378 & 144 & 112 & 4.79 & 30.365 \\
$\mathrm{PL}$ & 378 & 145 & 129 & 3.31 & 100.728 \\
\hline $\mathrm{AC}$ & 153 & 29 & 29 & 1.59 & 9.852 \\
$\mathrm{PR}$ & 153 & 62 & 48 & 7.30 & 20.842 \\
$\mathrm{PL}$ & 153 & 59 & 54 & 5.06 & 51.308 \\
\hline
\end{tabular}

We show the non-dominated points of the three clinical cases in Figs. 15 and 16. The generated non-dominated points clearly appear to be evenly distributed. We also note that the distance $d s$ of reference points and the distance of non-dominated points is measured in Gray, the unit of radiation dose, because all three objectives are measured in this unit. This unit has a clear meaning for radiotherapy planners. Since each non-dominated point represents a potential treatment plan, their distance can be interpreted as a measure of the difference of the plans. Radiotherapy planners may have a good idea what dose value would constitute recognisable significant difference in plans. Hence they should be able to set a desired value for the distance $d$ of non-dominated points, which then implies that $d s$ should be set to a value between $d / \sqrt{3}$ and $d$ to guarantee non-dominated points with a distance between $d$ and $\sqrt{3} d$. The value of $d$ then in turn determines the number of reference points to be used.
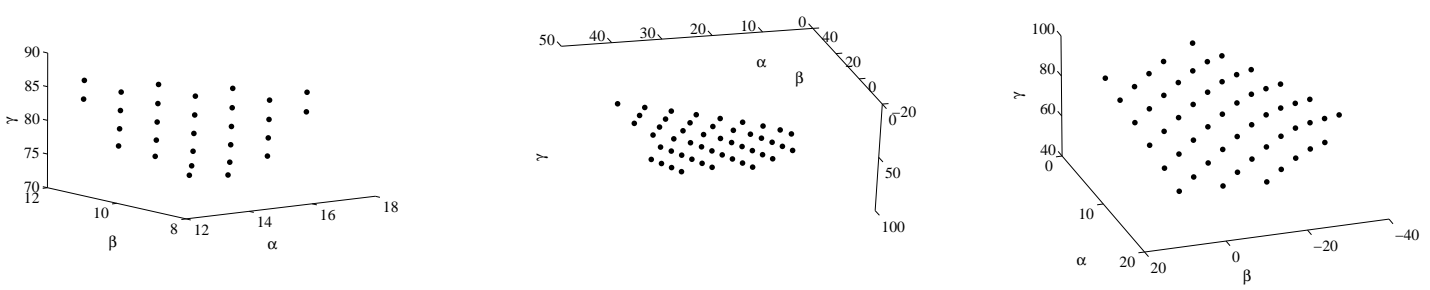

Figure 15: The non-dominated points generated for the AC, PR and PL cases (from left to right) when 153 reference points are used.

To conclude this section, we present numerical results for randomly generated MOLP problems. The examples are generated as follows. First, $l$ points $x^{i} \in \mathbb{R}^{p}, i=1, \ldots, l$ with $x_{1}^{i}, \ldots, x_{p-1}^{i}$ randomly distributed between 0 and 1 and $x_{p}^{i}=\left(x_{1}^{i}-1\right)^{2}+\ldots+\left(x_{n-1}^{i}-1\right)^{2}$ are generated. Next, the convex hull of the $l$ points $x^{i}, i=1, \ldots, l$ is constructed. Now, we let the convex hull be the feasible set $X$ in decision space, i.e., we use all facet defining inequalities of the convex hull as 

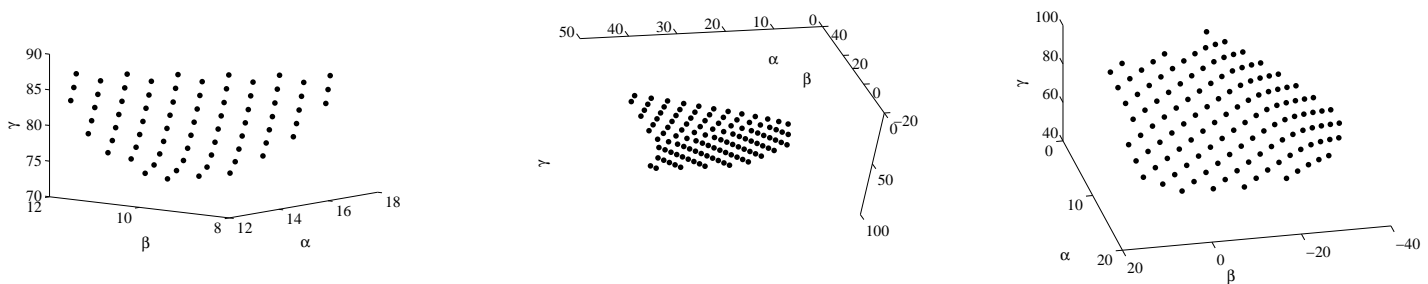

Figure 16: The non-dominated points generated for the AC, PR and PL cases (from left to right) when 378 reference points are used.

constraints of the MOLP. Finally, we set the objective matrix $C$ of the MOLP to be the identity matrix $I$. This procedure is employed in order to generate MOLPs with $p$-dimensional feasible set in objective space for which the non-dominated set has non-dominated facets. Since the randomly generated points are distributed on the lower-left part of the sphere $x_{n}=\left(x_{1}-1\right)^{2}+\ldots+\left(x_{n-1}-1\right)^{2}$, this does happen most of the time (always in our experiment). If, instead, we were to generate the coefficients of the constraint matrix, right hand side vector and objective function matrix of (1) randomly, it would quite often be the case that $Y$ and $Y_{N}$ have lower dimensions, in which case Theorem 5.2 tells us that the RNBI method may not work.

Ten examples for selected combinations of $l$ (number of points) and $p$ (number of objectives, which is equal to the number $n$ of variables) were solved for two different numbers of reference points (RP). The average number of constraints $m$ as well as the results, consisting of the average numbers of intersection points (IP), non-dominated points (NP), distance between two closest reference points $d s$ and the CPU time in seconds are listed in Table 2. We also show the generated non-dominated points and the feasible sets in objective space for two of the randomly generated examples with $(p, l, m)=(3,30,56)$ in Fig. 17. The one on the left has (RP,IP,NP, $d s)=(158$, $81,74,0.1273)$ and the one on the right $(82,40,37,0.2122)$.
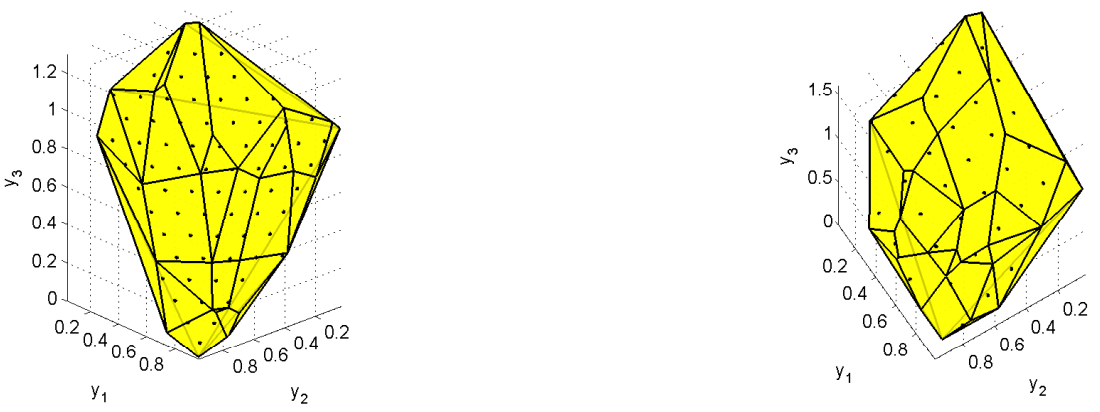

Figure 17: The generated non-dominated points and feasible sets in objective space for two of the randomly generated examples with $p=3$.

Table 2 shows that the CPU time clearly increases with the size of the MOLP (number of variables and constraints) and also the number of reference points, but as is usual in multi-objective 
Table 2: The number of objectives $p$, variables $n$, random points $l$, constraints $m$, reference points RP, intersection points IP on $Y$, non-dominated points NP, the distance $d s$ between two closest reference points and the CPU time in seconds.

\begin{tabular}{rrrrrrrr}
\hline$p=n$ & $l$ & $m$ & $\mathrm{RP}$ & $\mathrm{IP}$ & $\mathrm{NP}$ & $d s$ & $\mathrm{CPU}$ \\
\hline 3 & 30 & 56 & 82 & 40.8 & 39.3 & 0.1906 & 0.6108 \\
& & & 158 & 74.1 & 71.4 & 0.1375 & 1.2902 \\
\hline \multirow{2}{*}{4} & 40 & 195 & 340 & 82.4 & 74.9 & 0.2612 & 2.5516 \\
& & & 907 & 203.0 & 182.4 & 0.1938 & 6.3787 \\
\hline 5 & 50 & 748 & 947 & 85.3 & 72.7 & 0.3511 & 7.2225 \\
& & & 3627 & 297.6 & 246.9 & 0.2591 & 26.3532 \\
\hline \multirow{2}{*}{6} & 60 & \multirow{2}{*}{3041} & 1899 & 83.5 & 64.9 & 0.4339 & 17.6222 \\
& & & 10875 & 355.9 & 260.0 & 0.3197 & 130.5689 \\
\hline 7 & 70 & 12678 & 2852 & 57.3 & 46.7 & 0.4988 & 94.0288 \\
& & & 25345 & 360.4 & 255.5 & 0.3666 & 822.7798 \\
\hline \multirow{2}{*}{8} & \multirow{2}{*}{80} & 53239 & 3264 & 41.1 & 29.8 & 0.5687 & 447.4213 \\
& & & 47033 & 260.6 & 155.9 & 0.4191 & 6184.2201 \\
\hline
\end{tabular}

optimisation, the most significant impact on CPU time is the number of objective functions $p$. Nevertheless, the RNBI method can be applied to MOLPs with up to eight objective functions. Under the assumption of Theorem 5.2 it is guaranteed to generate an evenly distributed representative set of non-dominated points. It is also apparent that as $p$ increases, the number of reference points that do not generate an intersection point with $Y$ increases dramatically.

\section{Conclusion and Future Work}

In this paper, we have addressed the problem of generating a discrete representation of the non-dominated set of a multi-objective linear programme. We have proposed the revised normal boundary intersection method, which, by combining features of the normal boundary intersection method and the global shooting method overcomes drawbacks of both of these methods and generates an evenly distributed set of representative points. In fact, we have been able to prove a priori bounds on the uniformity level and the coverage error of the discrete representation under the assumption that the non-dominated faces of the feasible set in objective space are "big enough" relative to the distance of reference points. The RNBI method is the first method for which quality guarantees in terms of both coverage error and uniformity level have been proved. Moreover, numerical results on intensity optimisation problems from radiotherapy treatment planning and on randomly generated examples empirically confirm that the points of the discrete representation 
are indeed evenly distributed, and that it is applicable for MOLPs with up to eight objectives.

We have indicated in Section 6 above, that one of the problematic issues with the RNBI method is that many reference points lead to infeasible RNBI subproblems. Hence methods to decide whether (14) is feasible before solving it, would help eliminate unnecessary computation. This is a topic we will further investigate in the future. Moreover, we have seen that (14) may yield dominated intersection points with $Y$. In the case of $p=2$ objectives this can easily be avoided by replacing the anti-ideal point $y^{A I}$ by the nadir point $y^{N}$ in the construction of the reference plane. This makes it easy to determine which RNBI subproblems will yield a non-dominated point and the RNBI method becomes an algorithm that runs in time linear in the cardinality of the representation. However, the determination of the nadir point is itself a difficult problem for $p \geqq 3$, so other techniques to replace the anti-ideal point by a better upper bound on $Y_{N}$ are worth investigating.

In order to address more general problems, we will first generalise the method to MOLPs without the compactness assumption on $Y$, i.e., where $Y$ may be unbounded, with $Y_{N}$ either bounded or not. Another interesting extension to be addressed in future research is a variant of the RNBI method for convex multi-objective optimisation problems.

\section{Acknowledgements and Data Statement}

This work has been partially supported by Beijing Natural Science Foundation [grant number 4152034]; National Natural Science Foundation of China [grant number 81000650], [grant number 61370132] and National High-tech Research Development Program of China (863 Program) [grant number 2013AA040705]. More information on the data used in this paper can be found at DOI 10.17635/lancaster/researchdata/xx.

Baur, C. and Fekete, S. (2001). Approximation of geometric dispersion problems. Algorithmica, $\mathbf{3 0}(3), 451-470$.

Benson, H. P. (1998). An outer approximation algorithm for generating all efficient extreme points in the outcome set of a multiple objective linear programming problem. Journal of Global Optimization, 13(1), 1-24.

Benson, H. P. and Sayin, S. (1997). Towards finding global representations of the efficient set in multiple objective mathematical programming. Naval Research Logistics, 44(1), 47-67.

Das, I. and Dennis, J. E. (1998). Normal-boundary intersection: A new method for generating the pareto surface in nonlinear multicriteria optimization problems. SIAM Journal on Optimization, $\mathbf{8}(3), 631-657$.

Ehrgott, M. (2005). Multicriteria Optimization. Springer, Berlin Heidelberg, second edition. 
Ehrgott, M., Güler, c., Hamacher, H. W., and Shao, L. (2009). Mathematical optimization in intensity modulated radiation therapy. Annals of Operations Research, 175(1), 309-365.

Eusébio, A., Figueira, J., and Ehrgott, M. (2014). On finding representative non-dominated points for bi-objective integer network flow problems. Computers 6 Operations Research, 48, 1-10.

Faulkenberg, S. L. and Wiecek, M. M. (2010). On the quality of discrete representations in multiple objective programming. Optimization and Engineering, 11(3), 423-440.

Faulkenberg, S. L. and Wiecek, M. M. (2012). Generating equidistant representations in biobjective programming. Computational Optimization and Applications, 51(3), 1173-1210.

Greco, S., Ehrgott, M., and Figueira, J., editors (2016). Multiple Criteria Decision Analysis: State of the Art Surveys, Second Edition, volume 78 of International Series in Operations Research \& Management Science. Springer, New York, second edition.

Hancock, B. J. and Mattson, C. A. (2013). The smart normal constraint method for directly generating a smart Pareto set. Structural and Multidisciplinary Optimization, 48(4), 763-775.

Isermann, H. (1974). Proper efficiency and the linear vector maximum problem. Operations Research, 22, 189-191.

Ismail-Yahaya, A. and Messac, A. (2002). Effective generation of the Pareto frontier using the normal constraint method. In Proceedings of the $40^{\text {th }}$ Aerospace Sciences Meeting and Exhibit. Paper No. AIAA 2002-0178.

Logist, F. and Van Impe, J. (2012). Novel insights for multi-objective optimisation in engineering using normal boundary instersection and (enhanced) normalised normal constraint. Structural and Multidisciplinary Optimization, 45(3), 417-431.

Martinez, M., Sanchis, J., and Blasco, X. (2007). Global and well-distributed pareto frontier by modified normalized normal constraint methods for bicriterion problems. Structural and Multidisciplinary Optimization, 34(3), 197-209.

Messac, A. and Mattson, C. A. (2004). Normal constraint method with guarantee of even representation of complete pareto frontier. AIAA Journal, 42(10), 2101-2111.

Messac, A., Ismail-Yahaya, A., and Mattson, C. A. (2003). The normalized constraint method for generating the Pareto frontier. Structural and Multidisciplinary Optimization, 25(2), 86-98.

Motta, R. d. S., Afonso, S. M. B., and Lyra, P. R. M. (2012). A modified NBI and NC method for the solution of N-multiobjective optimization problems. Struct Multidisc Optim, 46(2), 239-259. 
Mueller-Gritschneder, D., Graeb, H., and Schlichtmann, U. (2009). A successive approach to compute the bounded pareto front of practical multiobjective optimization problems. SIAM Journal on Optimization, 20(2), 915-934.

Naccache, P. (1978). Connectedness of the set of nondominated outcomes in multicriteria optimization. Journal of Optimization Theory and Applications, 25(3), 459-467.

Rockafellar, R. T. (1970). Convex Analysis. Princeton University Press, Princeton NJ.

Ruzika, S. and Wiecek, M. M. (2005). Survey paper approximation methods in multiobjective programming. Journal of optimization theory and applications, 126(3), 473-501.

Sanchis, J., Martínez, M., Blasco, X., and Salcedo, J. V. (2008). A new perspective on multiobjective optimization by enhanced normalized normal constraint method. Structural and Multidisciplinary Optimization, 36(5), 537-546.

Sayin, S. (2000). Measuring the quality of discrete representations of efficient sets in multiple objective mathematical programming. Mathematical Programming, 87(3), 543-560.

Sayın, S. (2003). A procedure to find discrete representations of the efficient set with specified coverage errors. Operations Research, 51(3), 427-436.

Shao, L. and Ehrgott, M. (2007). Finding representative nondominated points in multiobjective linear programming. In 1st IEEE Symposium on Computational Intelligence in Multicriteria Decision Making, MCDM 2007, pages 245-252. IEEE.

Shao, L. and Ehrgott, M. (2008). Approximately solving multiobjective linear programmes in objective space and an application in radiotherapy treatment planning. Mathematical Methods of Operations Research, 68(2), 257-276.

Vaz, D., Paquete, L., Fonseca, C., Klamroth, K., and Stiglmayr, M. (2015). Representation of the non-dominated set in biobjective discrete optimization. Com puters $\&$ Operations Research, 63(1), 172-186.

White, D. (1991). The maximal dispersion problem and the "first point outside the neighbourhood heuristic". Computers \& Operations Research, 18, 43-50.

Yu, P. L. and Zeleny, M. (1975). The set of all nondominated solutions in linear cases and a multicriteria simplex method. Journal of Mathematical Analysis and Applications, 49, 430468. 